\title{
Path-centric On-demand Rate Adaptation for Mobile Ad Hoc Networks
}

\author{
Saehoon Kang*, Chansu $\mathrm{Yu}^{\dagger}$, Chita R. Das ${ }^{\ddagger}$ and Guohong Cao ${ }^{\ddagger}$ \\ *School of Engineering, Information and Communications University, Daejeon, Korea, 305-732, kang@icu.ac.kr \\ $\dagger$ Dept. of ECE, Cleveland State University, Cleveland, OH 44115, c.yu91@ csuohio.edu \\ $\dagger$ Dept. of CSE, The Pennsylvania State University, University Park, PA 16802, \{das, gcao\}@ cse.psu.edu
}

\begin{abstract}
Exploiting the multirate capability in mobile ad hoc networks (MANETs) is more complex than in single-hop WLANs because of the rate-distance and rate-hop count tradeoffs. This paper proposes Path-centric on-demand Rate Adaptation for MANETs (PRAM) protocol. A unique feature that sets PRAM apart from most of previous studies is its path-centric approach. While others focus on finding the best data rate for each link and offering a routing path as a collection of links at their best rates, PRAM finds the best data rate for a sourcedestination pair and then, dynamically adapts it based on path lifetime and other factors. Another distinctive feature of PRAM is that it can be seamlessly incorporated with an on-demand routing protocol. Extensive performance study based on ns-2 has demonstrated that PRAM achieves as much as $71.7 \%$ higher packet delivery ratio than fixed-rate cases $(6 \sim 54 \mathrm{Mbps})$ and as much as $43.2 \%$ higher than the multihop version of the wellknown ARF mechanism in a wide range of network scenarios. It is also shown that PRAM is capable of using a mixture of data rates in an adaptive manner.
\end{abstract}

Index Terms-Multirate adaptation, Mobile ad hoc networks, Path lifetime, IEEE 802.11, Spectral efficiency.

\section{INTRODUCTION}

With the availability of multirate capability in radio hardware and its inclusion in IEEE $802.11 \mathrm{a} / \mathrm{b} / \mathrm{g}$, research community is trying to figure out how to exploit this capability to improve the network performance [1]-[7], [9]-[14], [18]-[22]. Those efforts can largely be categorized according to whether they target on single-hop WLANs or multihop mobile ad hoc networks (MANETs). Exploiting the multirate capability in single-hop WLANs is relatively simple because node has only one communication partner (access point or AP) and no more than one communication can concurrently happen. Therefore, each node can individually optimize its data rate for the communication link to the AP [2], [3], [11], [12]. For example, in Auto-Rate Fallback (ARF) [12], a node lowers its data rate if it experiences consecutive transmission failures and increases its data rate upon a number of consecutive transmission successes.

On the other hand, exploiting multirate capability in MANETs is admittedly more complex because of mobility and multihop routing. First, note that a higher data rate is achieved by using a more spectrally efficient modulation scheme and thus, requires high signal-to-interference-noise ratio (SINR). This in turn, mandates a shorter communication range or a larger number of hops for a given source-destination node pair. Due to this rate-distance or rate-hop count tradeoff, high data rates are not always preferable. Second, due to the effect of inter-hop interference and almost identical interference range regardless of data rates, a high data rate brings in lower performance benefit than normally expected. Third, node mobility could break high-rate (short-range) links more easily, leading to a higher control overhead for maintaining active routing paths in a mobile environment.

A great deal of work on multirate adaptation has been reported in the context of multihop networks due to its potential in many interesting applications including wireless mesh networks [7], [9], [10], [13], [14], [18]-[21], [24]. Typically, they have concentrated on developing a rate-aware link cost which is then integrated with a multihop routing algorithm to find the path that minimizes the total cost.

However, most of previous studies target static networks and/or employ proactive routing algorithms such as Destination-Sequenced Distance Vector (DSDV) [16] as the underlying routing protocol [6]-[8], [27], [30]. Proactive routing algorithms could be a reasonable choice in static networks. However, they incur a high control overhead in a mobile multirate environment because a "larger volume" of route information must be exchanged "more frequently." In other words, each node is required to keep track of and exchange the status of all its links at all available data rates increasing the control overhead. More seriously, nodes need to exchange route information much more frequently than in conventional single-rate network, where the frequency is set to, for example, 12 seconds [26] under a typical MANET scenario. However, in a multirate environment, a high rate link could survive only for a shorter duration, demanding much more frequent information exchange to maintain the link status up-to-date.

This paper proposes Path-centric on-demand Rate Adaptation for MANETs (PRAM) protocol that is a rate adaptation scheme, seamlessly incorporated with an on-demand routing protocol, called the Ad-hoc On-demand Distance Vector (AODV) [17]. To the best of our knowledge, this is the first paper that addresses the design of multihop routing in a mobile, multirate environment. A unique feature that sets PRAM apart from most of the previous studies is its pathcentric approach. While others focus on finding the best data rate for each link to find an optimal path as a collection of links, PRAM finds an optimal data rate for a given sourcedestination pair. We believe this path-centric approach better fits in a multirate MANET than link-centric approaches be- 
cause of its significantly lower control overhead and a simpler route discovery and maintenance procedure. On the other hand, PRAM dynamically adapts the data rate of a routing path depending on its robustness or lifetime, which is in turn affected by several factors including source-destination distance, traffic, and mobility conditions. Also, PRAM allows a node to adapt its link rate individually based on the ARF principle [12].

Extensive performance study based on $n s-2$ network simulator [15] has been conducted to evaluate the performance of PRAM. It is compared against single-rate cases and the multihop version of ARF, called $M H-A R F$, where each node applies the ARF rule to each link individually. Our study indicates that the $6 \mathrm{Mbps}$ case performs better than the $54 \mathrm{Mbps}$ case when the network is sparse but it is the opposite when the network is dense. However, PRAM achieves the best performance in the entire range of node density. Packet delivery ratio (PDR) is improved as much as $72 \%, 68 \%, 46 \%, 33 \%, 22 \%, 15 \%$, $18 \%$, and $38 \%$ in comparison to fixed rate case of $6,9,12$, $18,24,36,48$ and $54 \mathrm{Mbps}$, respectively. In comparison to MH-ARF, it improves PDR as much as $34 \%$.

The rest of the paper is organized as follows: Section II discusses the background information and related work. Section III explains the proposed protocol, PRAM. It is followed by the presentation of ns-2-based experiment results in Section IV. Section V concludes the paper.

\section{BACKGROUND AND RELATED WORK}

\section{A. Background}

IEEE 802.11 and its extensions (IEEE $802.11 \mathrm{a} / \mathrm{b} / \mathrm{g}$ ) provide the capability to transmit data at different data rates. For example, IEEE 802.11a/g supports 6, 9, 12, 18, 24, 36, 48 and 54 Mbps. However, it is observed that performance does not improve linearly as the data rate increases even in a single-hop link. This is due to the rate-independent overhead at the PHY (PLCP preamble and header ${ }^{1}$ ) and MAC (SIFS, DIFS and backoff delay) layers [29]. Moreover, this overhead becomes a major part as the data rate increases because the transmission time of the actual payload decreases proportionally.

On the other hand, to increase the aggregate network throughput in a MANET, multiple concurrent communications should be simultaneously successful when they are outside of each other's interference range. It is dictated by two important parameters in wireless communications, called receive sensitivity and capture threshold. First, for a successful communication, the received signal power must be higher than the receive sensitivity in the presence of path loss over the distance. Second, the received signal power must be strong enough to overcome the influence of noise and interference from all other simultaneous transmissions, i.e., SINR must be higher than the capture threshold [23], [25].

${ }^{1}$ PLCP preamble and header at the PHY layer are transmitted at the lowest rate $(6 \mathrm{Mbps})$, while the payload is transmitted at a higher rate, which is specified in the control frame
Table I: Characteristics of an 802.11a multirate radio. (Transmit power: $6 \mathrm{dBm}$, radio propagation model: two-ray ground reflection with path loss exponent of 4.)

\begin{tabular}{|c||c|c||c|c|}
\hline $\begin{array}{c}\text { Data } \\
\text { rate } \\
\text { (Mbps) }\end{array}$ & $\begin{array}{c}\text { Receive } \\
\text { sensiti- } \\
\text { vity } \\
(\mathrm{dBm})\end{array}$ & $\begin{array}{c}\text { Communi- } \\
\text { cation } \\
\text { range } \\
\text { (meters) }\end{array}$ & $\begin{array}{c}\text { Capture } \\
\text { threshold }\end{array}$ & $\begin{array}{c}\text { Interfe- } \\
\text { rence } \\
\text { range } \\
(\text { meters })\end{array}$ \\
\hline \hline 6 & -82 & 238 & 6.02 & 575 \\
\hline 9 & -81 & 224 & 7.78 & 576 \\
\hline 12 & -79 & 200 & 9.03 & 536 \\
\hline 18 & -77 & 178 & 10.79 & 509 \\
\hline 24 & -74 & 150 & 17.04 & 550 \\
\hline 36 & -70 & 119 & 18.80 & 470 \\
\hline 48 & -66 & 95 & 24.05 & 484 \\
\hline 54 & -65 & 89 & 24.56 & 455 \\
\hline
\end{tabular}

Table $\mathrm{I}^{2}$ shows the two parameters at different data rates for a typical 802.11a radio device [24]. A high data rate communication requires a higher receive sensitivity or a shorter communication distance. Also, it requires a higher capture threshold, which means more vulnerability to interference. In other words, although a high rate communication is rendered short-distanced, it still requires the interference range as large as low-rate communications. The parameters in Table I are used in our simulation study detailed in Section IV.

\section{B. Related Work}

The research on multirate adaptation targets either singlehop wireless LANs [1], [10]-[12], [18] or multihop networks.

Auto-Rate Fallback (ARF) [12] is the first multirate algorithm, which is sender-based, and was designed to optimize the application throughput in WaveLan II devices. The basic idea of ARF is to use a higher rate upon consecutive successful transmissions and to fall back to a lower rate after a number of consecutive transmission failures. Note that a transmission is judged as a success or a failure based on the ACK signal from the receiver.

Receiver-Based Auto Rate (RBAR) [10] is a receiver-based multirate algorithm. It assumes to use the RTS/CTS handshaking. Upon receiving an RTS frame, the receiver estimates the channel quality based on the Signal to Interference and Noise Ratio (SINR) of the received RTS frame and then determines the best data rate that the transmitter must use. The estimated optimal rate is then sent back to the sender by piggybacking in the CTS packet.

A great deal of work on multirate adaptation has been reported in the context of multihop networks [7]-[10], [13], [14], [18]-[21], [24] due to its potential to greatly improve the network throughput. They can be categorized as proactive or on-demand depending on the routing algorithm used. With a proactive multirate algorithm, each node maintains link costs to each of its neighbors while taking the multirate capability into account. Transition from a single-rate to the corresponding multirate algorithm is conceptually straightforward but at

\footnotetext{
${ }^{2}$ Communication range in the Table is computed as the distance at which the received signal power is equal to the receive sensitivity at that rate. Interference range in the table refers to the distance at which the SIR at the receiver is equal to the capture threshold when the sender-receiver separation coincides the communication range.
} 
the cost of very high, periodic control overhead due to the multiplicity of route information at multiple data rates.

Sheu et al. proposed Multi-Rate and Multi-Range Routing Protocol ( $\left.\mathrm{MR}^{2} \mathrm{RP}\right)$, in which each node runs a distributed Bellman-Ford routing algorithm to find the optimal (smallest delay) routing path based on a connectivity matrix [27]. In the matrix, each element corresponds to the delay between two nodes calculated based on the highest data rate supported plus the MAC-layer delay. Awerbuch et al. suggested a similar approach based on a proactive routing protocol, DSDV, and a new metric called Medium Time Metric (MTM) [7]. It is designed to minimize the total medium time consumed sending packets from a source to destination. Zhai and Fang proposed another new metric, called the bandwidth distance product (BDiP), which uses both channel rate and hop distance to determine the best candidate of the next hop [24].

However, the above-mentioned protocols are based on proactive routing algorithms and are known to incur a high control overhead in a mobile environment. Meanwhile, srcr [8] and MR-LQSR [6] use both proactive (link state) and ondemand (DSR [30]) algorithms but they target static wireless mesh networks.

\section{PATH-CENTRIC ON-DEMAND RATE ADAPTATION FOR MANETS (PRAM)}

This section presents a multirate adaptation algorithm, called Path-centric on-demand Rate Adaptation for MANETs (PRAM). In PRAM, a source node floods a RREQ to find a routing path as in a typical on-demand routing protocol such as DSR and AODV. Unique to PRAM is to dictate a data rate for the RREQ and to enforce every intermediate node to use the same rate when forwarding the RREQ. It may discover suboptimal routing paths at the benefit of simplicity and lower control overhead. Key design issues in PRAM are how to determine the data rate for the RREQ and when and how to increase or decrease the data rate.

\section{A. Multirate adaptation in PRAM}

On-demand routing algorithms such as AODV use RREQs to find routing paths. Therefore, it is important to use an appropriate data rate when broadcasting a RREQ. For example, if RREQs are forwarded at the lowest rate (6 Mbps), the discovered routing path will consist of long-range links, the communication over which may not be successful at higher data rates. In other words, the data rate for RREQs in essence limits the maximum feasible data rate for the links of the discovered routing paths. An alternative approach is to allow each intermediate node to use a different rate when it forwards a RREQ. For that matter, each node needs to maintain the best data rate for each of its links considering the communication environment in its local neighborhood. However, it is not straightforward for the node to determine the best rate for a broadcast packet such as RREQ.

Optimal data rate for a node pair: PRAM is a pathcentric adaptation scheme. A source node determines the data rate for a path to the destination and broadcasts a RREQ at that rate. Intermediate nodes are obliged to use the same data rate when forwarding the RREQ. When a data rate is too high, there could be a problem of connectivity. On the other hand, when a data rate is too low, the network is unable to realize its maximum capacity allowed by the radio hardware. Therefore, essential to the PRAM protocol is for a source node to determine the destination-specific data rate. Our approach in PRAM is (i) to determine the initial data rate based on the estimation of source-destination distance and (ii) to adapt it based on the path lifetime.

First (initialization), when a source node does not have any information about the destination node, it will try the medium data rate $(24 \mathrm{Mbps})$. However, when the source has communicated with the destination in the recent past, it can use the same data rate as an initial try. Second (adaptation), during the communicating with the destination, the source will adapt the rate as follows; (i) If a routing path survives longer than a certain threshold at the current data rate $\left(r_{i}\right)$, the source node may be better off by increasing the rate because the path is considered stable enough to support a higher data rate. The source node will send a probe RREQ (pRREQ) message at a higher data rate $\left(r_{i+1}\right)$. Note that pRREQ has the same packet format and is handled the same way as RREQ. Probe packet is not a new idea and has been used in single-hop WLANs [8], [12]. When a RREP arrives at the source, it will decide whether it is beneficial to switch to the newly discovered routing path (at $r_{i+1}$ ) or not based on the metric, which we will discuss shortly. (ii) If a routing path breaks earlier than the threshold time, the source node will try to find an alternative routing path at the current data rate $\left(r_{i}\right)$. (iii) If the destination is found unreachable due to the limited communication range at the current data rate, the source will broadcast a RREQ again at a decreased rate $\left(r_{i-1}\right)$.

Data rate selection metric: In conventional MANET routing algorithms, hop count is used as a metric to compare the candidate routing paths. However, in a real-world deployment of multihop networks, it has been observed that a shortest path is not necessarily the optimal path [4], [6], [8], [28]. In a multirate, mobile environment, it becomes even more complicated. The most agreeable metric would be the channel resource spent to deliver a packet end-to-end. In other words, the maximum aggregate throughput can be obtained by utilizing as little channel resource $\left(\mathrm{m}^{2} \cdot \mathrm{sec} / \mathrm{bit}\right)$ as possible so that the remaining resource can be utilized for other communications.

The metric used in PRAM is the product of channel space and time (PST), and is aggregated by using RREP messages with the help from the MAC layer. When a RREP message is delivered from a destination to a source at a data rate $r_{i+1}$, each intermediate node calculates the PST and piggybacks the accumulated PST in the RREP message. Upon receiving a RREP, the source node obtains the aggregated PST, which can then be used to compare with that at the current rate $r_{i}$. The channel time can be roughly estimated by a simple calculation based on the data rate and packet size. One additional consideration is to take the number of retransmissions 
at the MAC layer into account as in [4], [19]. For that matter, PRAM requires interactions between the MAC and routing layer, leading to a cross-layer design. The channel space consumed per transmission is approximated as $\left(\pi \cdot I R^{2}\right)$, where $I R$ denotes the interference range, shown in Table I. PRAM decides the optimal data rate that minimizes the aggregated PST.

Link rate optimization: It is oftentimes beneficial to use a higher rate than the source-dictated rate for a particular link. It can help in a temporarily congested area where the fast processing of a packet could allow other nodes to utilize the shared channel. Each node will maintain an optimal data rate for a particular link as in ARF [12]. More specifically, when a node transmits 10 packets successfully for a particular neighbor at the specified data rate $\left(r_{i}\right)$, it increases the data rate for the neighbor to $r_{i+1}$. When the rate is increased, the first transmission must succeed. Otherwise, the rate immediately falls back to $r_{i}$. However, it does not go below the sourcedictated data rate. Note that this optimization is implemented at the MAC layer and each node maintains a data rate for each of its neighbors to apply the ARF rule independently.

\section{B. Implementation of PRAM}

Fig. 1 shows the overview of the PRAM protocol based on the AODV algorithm [17]. Key changes to the original AODV include (i) routing table entries augmented by data rates, (ii) new control packet, pRREQ, which is sent to discover a new route at a higher data rate, and (iii) RREP packet piggybacked with PST to help determine the best data rate for a routing path. All the data and control packets are transmitted at the source-dictated data rate, but the source dynamically adapts it based on the path lifetime.

Another important consideration in the PRAM protocol is the decision of the unreachability of the destination. In the original AODV [17], if network-wide broadcasting of RREQ fails four times consecutively, the destination is considered unreachable and the source drops all the packets. In PRAM, when the source fails to receive a RREP for a network-wide flood of RREQ, it will decrease the data rate of the next broadcasting of a RREQ until it reaches the lowest data rate available (i.e., 6Mbps). This is because the destination might not be reachable at a higher rate.

Changes in the 802.11 MAC protocol for implementing the PRAM protocol are: (i) When a MAC layer passes a received packet to the network layer, it informs the data rate at which it received. (ii) When the network (routing) layer passes a packet to the MAC for transmission, it specifies the transmission rate for the packet. (iii) For implementing the localized link rate optimization, each node maintains the status of the links to its communicating neighbors. As in ARF [12], it increases and decreases the data rate depending on the recent history of communication successes and failures over the specific link.

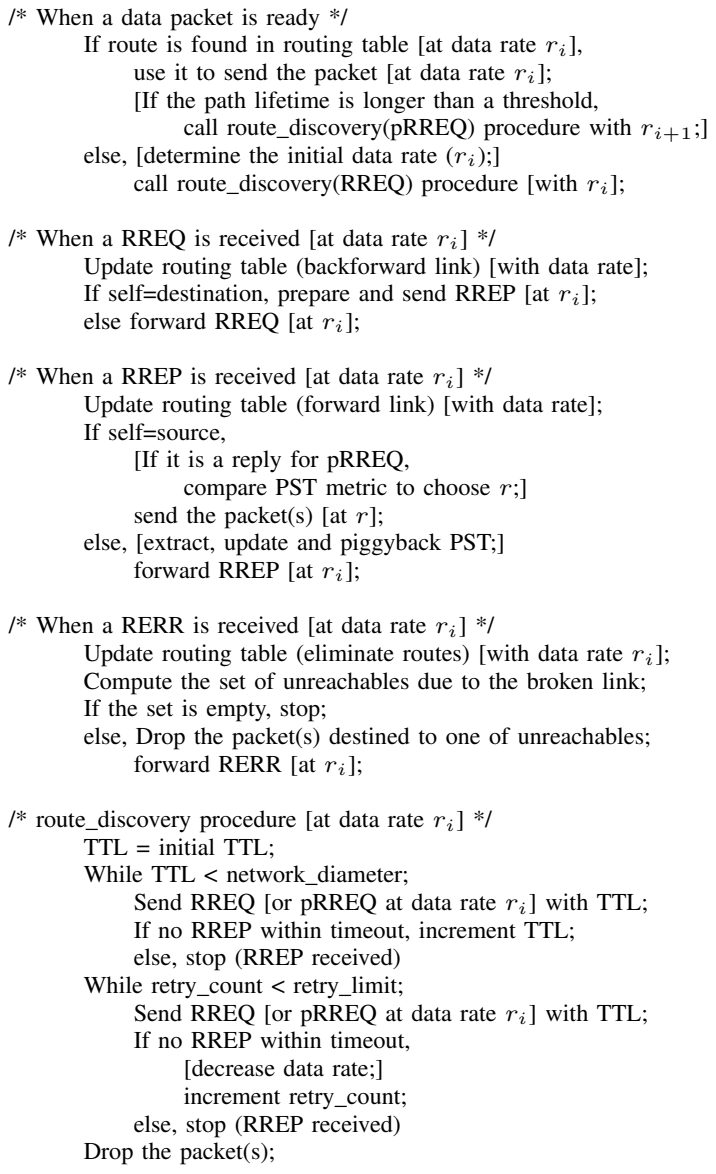

Figure 1: Overview of the PRAM protocol based on AODV. (All the changes to the original AODV are denoted within brackets.)

\section{Performance Evaluation}

\section{A. Simulation environment}

The performance of PRAM is evaluated using ns-2 [15], which simulates node mobility, a realistic physical layer, radio network interfaces, and the 802.11 MAC protocol. Our evaluation is based on the simulation of $20 \sim 140$ mobile nodes located in an area of $1500 \times 300 \mathrm{~m}^{2}$. The data traffic simulated is constant bit rate (CBR) traffic. 20 CBR sessions are simulated at the packet rate of 20 packets/second. Random waypoint mobility model [30] is used in our experiments with the minimum and maximum node speed of $1 \mathrm{~m} / \mathrm{s}$ and $5 \mathrm{~m} / \mathrm{s}$, and a pause time of 0 second. With this mobility model, a node travels (between 1 and $5 \mathrm{~m} / \mathrm{s}$ ) towards a randomly selected destination in the network. After the node arrives at the destination, it travels towards another randomly selected destination. Simulation time is 300 seconds for each run.

The aforementioned simulation parameters are typical in many previous studies on MANET including [26] except that the traffic intensity and the number of nodes $(N)$ are higher than normal. The traffic intensity of 20 sessions with 20 packets/second each could be overwhelming at $6 \mathrm{Mbps}$, but it can be reasonably handled at $54 \mathrm{Mbps}$. $N$ is as many as 140 in our simulation study because a high node density is needed for communications at high data rates. In fact, we are 


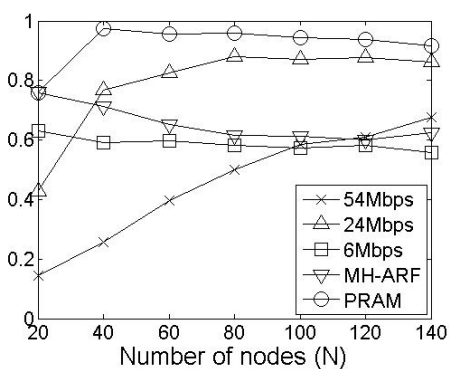

(a) Packet delivery ratio

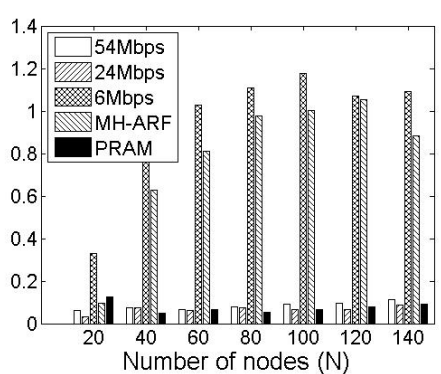

(b) Average packet delay (seconds)

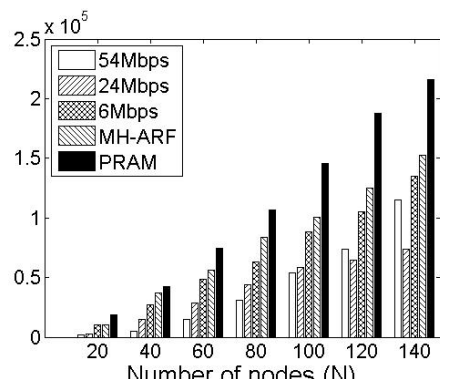

Number of nodes (N)
(a) Number of RREQs/pRREQs

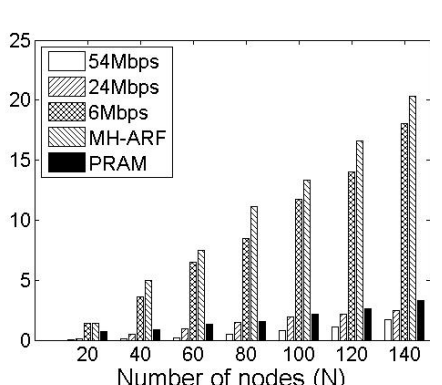

(b) Channel resource consumption due to RREQs/pRREQs $\left(\mathrm{m}^{2} \cdot \mathrm{sec} \cdot 10^{6}\right)$

Figure 2: Performance comparison. (PRAM achieves the best performance in the wide range of $N$ in (a). MH-ARF improves over the $6 \mathrm{Mbps}$ case but is not comparable to PRAM. In (b), low rate cases exhibit high packet delay due to their slow communication speed. The low packet delay in high rate cases is partly due to their low PDR. PRAM provides low packet delay without deteriorating the PDR.)

particularly interested in the performance variation with the varying number of nodes in the network.

We compare ten different schemes: fixed data rates of $6 \sim$ 54Mbps, multihop version of ARF (MH-ARF), and PRAM. In MH-ARF, each node maintains the best rate for each of its neighbors by applying the ARF rule separately. The ARF rule is explained in Section II-B. On the other hand, the fixed-rate cases denote the scenario, where all control and data packets are transmitted at the specified data rate. In a sparse network (e.g., 20 nodes in the network), we expect the 54 Mbps case would suffer the most because of the connectivity problem. But, it will become advantageous as $N$ increases. We have not simulated multirate, proactive routing algorithms in this paper because (i) their performance is not comparable due to high control overhead in a mobile environment, and (ii) direct comparison of proactive and on-demand (multirate) algorithms is not fair unless protocol parameters are carefully chosen.

Performance metrics are packet delivery ratio (PDR) and average packet delay. Since PRAM is implemented mostly at the routing layer, we also measured the total control overhead at the routing layer (RREQ/pRREQs, RREPs and RERRs). For PRAM, statistics on the mixture of data rates used are also presented to understand the adaptive behavior of the PRAM protocol.

\section{B. Simulation results}

Fig. 2 compares PDR and average packet delay of the fixed rate cases, MH-ARF and PRAM. For clarity, we show only three (54Mbps, 24Mbps and 6Mbps) out of the eight fixed rate cases. Fig. 2(a) shows the PDR versus $N$. The $54 \mathrm{Mbps}$ case does not function well as shown in the figure, particularly with a small $N$. This is mainly due to the lack of end-to-end connectivity. However, its performance increases rapidly as $N$ increases. A similar pattern is observed for other high data rates. In the $6 \mathrm{Mbps}$ case, the PDR is the highest when $N$ is 20 and decreases as $N$ increases. What matters at $6 \mathrm{Mbps}$ is not the end-to-end connectivity, but the traffic intensity because control traffic (broadcast of RREQs) increases with $N$. MH-ARF performs better than the $6 \mathrm{Mbps}$ case but the corresponding improvement is not significant.

Figure 3: Control overhead. (The number of RREQs is the highest in PRAM in (a). However, the channel resource consumption, measured in $m^{2} \cdot s e c$, is relatively small as shown in (b). Note that RREQs/pRREQs are responsible for the major part of routing layer control overhead. RREPs and RERRs take less than $10 \%$ of the total control overhead.)

Note that PRAM achieves the best performance in the entire range of $N$ as shown in Fig. 2(a). The main reason behind the superior performance of PRAM is that it uses a combination of all available data rates to maximize the network performance.

Fig. 2(b) shows the average packet delay versus $N$. The $6 \mathrm{Mbps}$ case has the highest packet delay because of its slow packet transmission speed. PRAM performs on par with the high rate cases as shown in the figure. However, the low packet delay for high rate cases does not represent their true performance because their PDR is low, and the computation of the average packet delay does not take the lost packets into account. On the other hand, PRAM's low packet delay in Fig. 2(b) demonstrate its exceptional performance because its PDR is the highest as shown in Fig. 2(a). The packet delay of MHARF is lower than the $6 \mathrm{Mbps}$ case but, again, it is still much higher than the proposed PRAM protocol.

A caveat of PRAM is its additional control overhead at the routing layer because it broadcast RREQs to use a higher-rate path whenever the preset condition is satisfied. More RREQs (pRREQs) incur more RREPs. The number of RERRs could be higher too because the new path at a higher rate could break more easily. Since RREQs are the dominant routing layer control overheads in AODV as discussed in [26] as well as observed in our simulation study, we compare the number of RREQs only. In Fig. 3(a), it is observed that the number of RREQs increases as $N$ increases. This is expected because RREQs are broadcast messages and every node will forward it once as long as its TTL is large enough (network-wide broadcast). Another clear trend is that PRAM produces the largest number of RREQs as discussed above. It is noted, however, that the number of RREQs does not offer the correct level of overhead because a RREQ at 6Mbps consumes more channel resource, or PST mentioned in Section III-A, than that at 54Mbps. Fig. 3(b) compares the PST consumed by RREQs during the simulation runs. Although the number of RREQs in PRAM is the largest in Fig. 3(a), the corresponding PST is relatively small compared to others. In fact, the $6 \mathrm{Mbps}$ case consumes the second largest PST because each RREQ takes a longer channel time. 


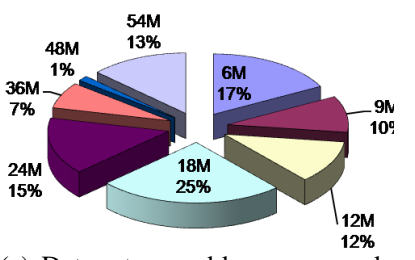

(a) Data rates used by source node (20-node network)

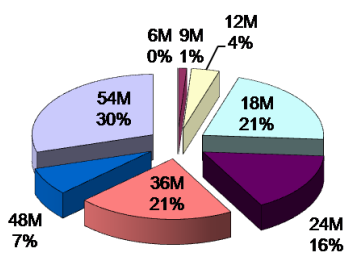

(b) Data rates used by source node (140-node network)
Figure 4: Statistics of data rate in PRAM. (In the 20-node network, low rate communications dominate the network as shown in (a). More nodes in 140-node network have a chance to use high data rates as in (b).)

In order to understand how PRAM improves the network performance, we collect statistics about data rate of the discovered routing paths. Figs. 4(a) and 4(b) show the mixture of data rates used in 20- and 140-node networks, respectively. In the 20 -node network, the mixture is $17 \%, 10 \%, 12 \%, 25 \%, 15 \%$, $7 \%, 1 \%$ and $13 \%$ for $6 \sim 54 \mathrm{Mbps}$. About $80 \%$ of routing paths use $6 \sim 24 \mathrm{Mbps}$. On the other hand, in the 140-node network, the mixture becomes $0 \%, 1 \%, 4 \%, 21 \%, 16 \%, 21 \%, 7 \%$, and $30 \%$, and more than $95 \%$ of routing paths use 18 54 Mbps. In other words, the PRAM protocol discovers routing paths at lower rates when network is sparse and at higher rates when network is dense.

In summary, PRAM uses a good mixture of data rates for routing paths depending on their source-destination separation and node density and thus, utilizes minimal channel resource to significantly improve the network's packet delivering capability without degrading the packet delay.

\section{CONCLUSION AND FUTURE WORK}

This paper discusses complex tradeoffs in a mobile environment and presents a new multirate adaptation protocol in the context of 802.11 MAC and AODV without incurring a high control overhead. A key idea of the proposed PRAM protocol is a path-centric or top-down approach. In other words, it finds an optimal data rate for a path and dynamically adapts it based on link/path lifetime. Extensive performance study based on ns-2 network simulator shows that PRAM performs better than fixed-rate scenarios (6 54Mbps) as well as the multihop version of ARF (MH-ARF) in terms of PDR and packet delay in the entire range of node density owing to the adaptive behavior of PRAM under different network conditions.

PRAM opens many interesting directions of research to pursue. For example, we are currently investigating the application of PRAM in multi-radio, multi-channel wireless mesh networks. Second, the performance and control overhead of PRAM will be compared against proactive algorithm-based multirate adaptation protocols [7], [24], [27]. Third, accurate estimation of path lifetime in real mobile environment and its impact on the effectiveness of the PRAM protocol is another interesting research subject.

\section{REFERENCES}

[1] A. Akella, G. Judd, P. Steenkiste, S. Seshan. Self Management in Chaotic Wireless Deployments. ACM MobiCom, 2005.

[2] J. Choi, K. Park, C.-K. Kim. Cross-Layer Analysis of Rate Adaptation, DCF and TCP in Multi-Rate WLANs. IEEE INFOCOM, 2007.

[3] S. Choi, K. Park, C.-K. Kim. On the performance characteristics of WLANs: revisited. ACM SIGMETRICS, 2005.

[4] D. S. J. De Couto, D. Aguayo, J. Bicket, R. Morris. A high-throughput path metric for multi-hop wireless routing. ACM MobiCom, 2003.

[5] M. Heusse, F. Rousseau, G. Berger-Sabbatel, A. Duda. Performance anomaly of $802.11 \mathrm{~b}$. IEEE INFOCOM, 2003.

[6] R. Draves, J. Padhye, B. Zill. Routing in Multi-Radio, Multi-Hop Wireless Mesh Networks. ACM MobiCom, 2004.

[7] B. Awerbuch, D. Holmer, H. Rubens. The Medium Time Metric: High Throughput Route Selection in Multi-rate Ad Hoc Wireless Networks. Mobile Networks and Applications, Springer, 11, 2006, 253-266.

[8] J. Bicket, D. Aguayo, S. Biswas, R. Morris. Architecture and Evaluation of an Unplanned 802.11b Mesh Network. ACM MobiCom, 2005.

[9] V. Gambiroza, B. Sadeghi, E. W. Knightly. End-to-end performance and fairness in multihop wireless backhaul networks. ACM MobiCom, 2004.

[10] G. Holland, N. Vaidya, P. Bahl. A Rate-Adaptive MAC Protocol for Multi-Hop Wireless Networks. ACM MobiCom, July 2001.

[11] Z. Ji, Y. Yang, J. Zhou, M. Takai and R. Bagrodia. Exploiting Medium Access Diversity in Rate Adaptive Wireless LANs. ACM MobiCom, 2004.

[12] A. Kamerman, L. Monteban. WaveLAN-II: A High-performance Wireless LAN for the Unlicensed Band. Bell Labs Technical Journal, 1997.

[13] T.-Y. Lin, J. C. Hou. Interplay of Spatial Reuse and SINR-Determined Data Rates in CSMA/CA-Based, Multi-Hop, Multi-Rate Wireless Networks. IEEE INFOCOM, 2007.

[14] H. Ma, S. Roy. Simple and Effective Carrier Sense Adaptation for Multi-rate Ad-Hoc MESH Networks. Intl. Workshop Wireless MESH Networks and Applicns., 2006.

[15] ns-2 Network Simulator, http://www.isi.edu/nsnam/ns/

[16] C. E. Perkins, P. Bhagwat. Highly Dynamic Destination-Sequenced Distance-Vector Routing (DSDV) for Mobile Computers. Computer Communications Review, 1994, 234-244.

[17] C. E. Perkins, and E. Royer. Ad-hoc On-Demand Distance Vector Routing. IEEE Workshop on Mobile Computing Systems and Applications, 1999, 90-100.

[18] B. Sadeghi, V. Kanodia, A. Sabharwal, E. Knightly. Opportunistic media access for multirate ad hoc networks. ACM MobiCom, 2002.

[19] S. H. Y. Wong, H. Yang, S. Lu, V. Bharghavan. Robust rate adaptation for 802.11 wireless networks. ACM MobiCom, 2006.

[20] X. Yang, N. Vaidya. Spatial Backoff Contention Resolution for Wireless Networks. Technical Report, UIUC, March 2006.

[21] S. Zhao, Z. Wu, A. Acharya, D. Raychaudhuri. PARMA: A PHY/MAC Aware Routing Metric for Ad-Hoc Wireless Networks with Multi-Rate Radios. IEEE WoWMoM, 2005.

[22] I. Tinnirello, S. Choi. Temporal fairness provisioning in multirate contention-based 802.11e WLANs. IEEE WoWMoM, 2005.

[23] C. Yu, K. G. Shin, L. Song. Maximizing Communication Concurrency via Link-Layer Packet Salvaging in Mobile Ad Hoc Networks. IEEE Trans. Mobile Computing, 6(4), Apr. 2007.

[24] H. Zhai, Y. Fang. Physical Carrier Sensing and Spatial Reuse in Multirate and Multihop Wireless Ad Hoc Networks. IEEE INFOCOM, 2006.

[25] M. Zorzi, R. Rao. Capture and retransmission control in mobile radio. IEEE Journal on Selected Areas in Communications, 12(8), 1994.

[26] J. Broch, D. A. Maltz, D. B. Johnson, Y.-C. Hu and J. Jetcheva. Performance Comparison of Multi-Hop Wireless Ad Hoc Network Routing Protocols. ACM MobiCom, 1998.

[27] S.-T. Sheu, Y. Tsai, J. Chen. MR2RP: The Multi-Rate and Multi-Range Routing Protocol for IEEE 802.11 Ad Hoc Wireless Networks. Wireless Networks, 9(2), 2003, 165-177.

[28] D. S. J. De Couto, D. Aguayo, B. A. Chambers, R. Morris. Performance of Multihop Wireless Networks: Shortest Path is Not Enough. The First Workshop on Hot Topics in Networks (HotNets-I), 2002.

[29] IEEE 802.11-1999, Local and Metropolitan Area Network, Part 11: Wireless LAN Medium Access Control and Physical Layer Spec.

[30] D. Johnson, and D. Maltz. Dynamic Source Routing in Ad-Hoc Wireless Networks. Ch. 5, Mobile Computing, edited by T. Imielinski, and H. Korth. Kluwer Academic Publishers, 1996, 153-181. 